Musées, Patrimoine et Culture scientifiques et techniques

$155 \mid 2014$

septembre-octobre 2014

\title{
Les parcours audioguidés : déambulation des publics et médiation sonore embarquée
}

\section{Sophie Deshayes}

\section{OpenEdition \\ Journals}

Édition électronique

URL : http://journals.openedition.org/ocim/1448

DOI : $10.4000 /$ ocim. 1448

ISSN : 2108-646X

Éditeur

OCIM

Édition imprimée

Date de publication : 1 septembre 2014

Pagination : 29-35

ISSN : 0994-1908

Référence électronique

Sophie Deshayes, «Les parcours audioguidés : déambulation des publics et médiation sonore

embarquée », La Lettre de I'OCIM [En ligne], 155 | 2014, mis en ligne le 01 septembre 2016, consulté le 19 avril 2019. URL : http://journals.openedition.org/ocim/1448 ; DOI : 10.4000/ocim.1448

Ce document a été généré automatiquement le 19 avril 2019

Tous droits réservés 


\title{
Les parcours \\ audioguidés : déambulation des publics et médiation sonore embarquée
}

\author{
Sophie Deshayes
}

1 Disponibles depuis le début des années 1980, les audioguides sont restés peu investis au musée jusqu'au tournant des années 2000, période où les technologies numériques supplantent les systèmes de guidage par infrarouge. Plusieurs facteurs concourent désormais à leur banalisation comme outil d'aide à la visite muséale. Un des premiers facteurs est lié au développement du tourisme culturel. Un autre facteur relève du marché des technologies mobiles. Enfin, résultat de nos propres analyses, un troisième facteur déterminant correspond au développement des pratiques culturelles médiatiques intensifiant une culture de l'écoute. 
Audioguide proposé en 2003 au Museon Arlaten, précurseur - en France - de l'usage du multimédia mobile comme outil d'aide à la visite des collections permanentes.

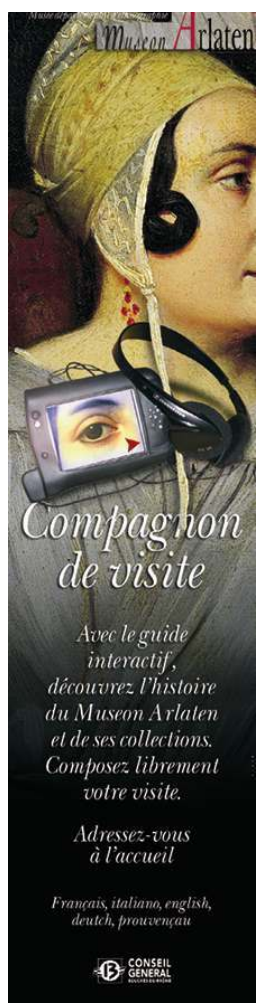

(C) Réalisation graphique Alyen

2 L'accueil des touristes, manne de fréquentation toujours renouvelée, s'est peu à peu imposé comme préoccupation principale dans le mode de développement de la relation aux publics. Outil de traduction multilingue, l'audioguide accompagne de fait ce développement. Mais ce n'est pas seulement la multiplication des versions en langues étrangères qui explique la généralisation de l'audioguidage au musée. Ces dernières années, les efforts ont porté sur la conception de programmes de contenus de médiation, destinés aux publics francophones, programmes traduits et déclinés ensuite en versions étrangères dans un souci plus ou moins abouti d'adaptation linguistique.

Amorcé au début des années 2000, l'essor des technologies mobiles constitue un autre facteur puissant: technologies sans fil, réseaux de télécommunication, une nouvelle industrie voit le jour autour des premiers PDA (ordinateur de poche), des baladeurs numériques et des téléphones mobiles communiquant. Pour leur commercialisation en masse, constructeurs et promoteurs de ces nouvelles technologies ont besoin de convaincre de l'intérêt de leur appropriation. L'alliance avec les industries du contenu sera en cela décisive. Les applications éducatives et culturelles sont envisagées comme un levier d'acceptation sociale de nouvelles performances technologiques dont on se passait bien jusqu'alors. Ce phénomène n'est pas nouveau, concernant les "machines à communiquer", ainsi dénommées par Pierre Schaeffer en 1970. Les travaux de Jacques Perriault puis ceux de Pierre Mœglin attestent bien de la manière dont les développements des techniques audiovisuelles sont passées en leur temps par une phase d'exploration des possibles auprès des acteurs de la sphère éducative : "vulgarisateurs, animateurs et pédagogues sont parmi d'autres, les "haut-parleurs" de l'invention (...)" 
(Perriault, 1989). Il serait donc tout à fait erroné de concevoir le monde des musées comme celui de l'immobilisme, du conservatisme paralysant que la technique viendrait bousculer, faire bouger. C'est au contraire une dynamique de mouvement permanent qui anime la communauté muséale depuis ses origines.

4 L'industrie des technologies mobiles se sert aujourd'hui d'une tradition d'innovation pédagogique au musée pour asseoir son développement sur la base d'une offre de contenus attractifs. Ainsi, on ne compte plus les offres en ligne de parcours destinées à la balado-diffusion. En contribuant à la production de balades sonores téléchargeables, les savoirs et savoir-faire du musée s'affranchissent des espaces intérieurs : "hors les murs" le périmètre de la visite s'élargit aux espaces publics où des objets patrimoniaux dignes d'intérêt sont signalés et commentés.

5 Nous laisserons ici de côté ces parcours audioguidés hors les murs, pour revenir à l'expérience de visite au musée et en tirer quelques enseignements pour penser le rapport entre déambulation des publics et besoins de médiation embarquée. En recentrant l'attention sur le sens de l'usage et les enjeux de médiation propres au musée, il est possible de contrer l'assignation de l'évidence technologique par des démarches de conception réfléchie du point de vue des publics et de leurs manières de faire pour en tirer le meilleur parti.

Au musée de la Civilisation à Québec, I'utilisation d'un audioguide type iPod, a permis d'offrir au public une visite commentée autonome de l'exposition Copyright humain, consacrée à la pensée humaine.

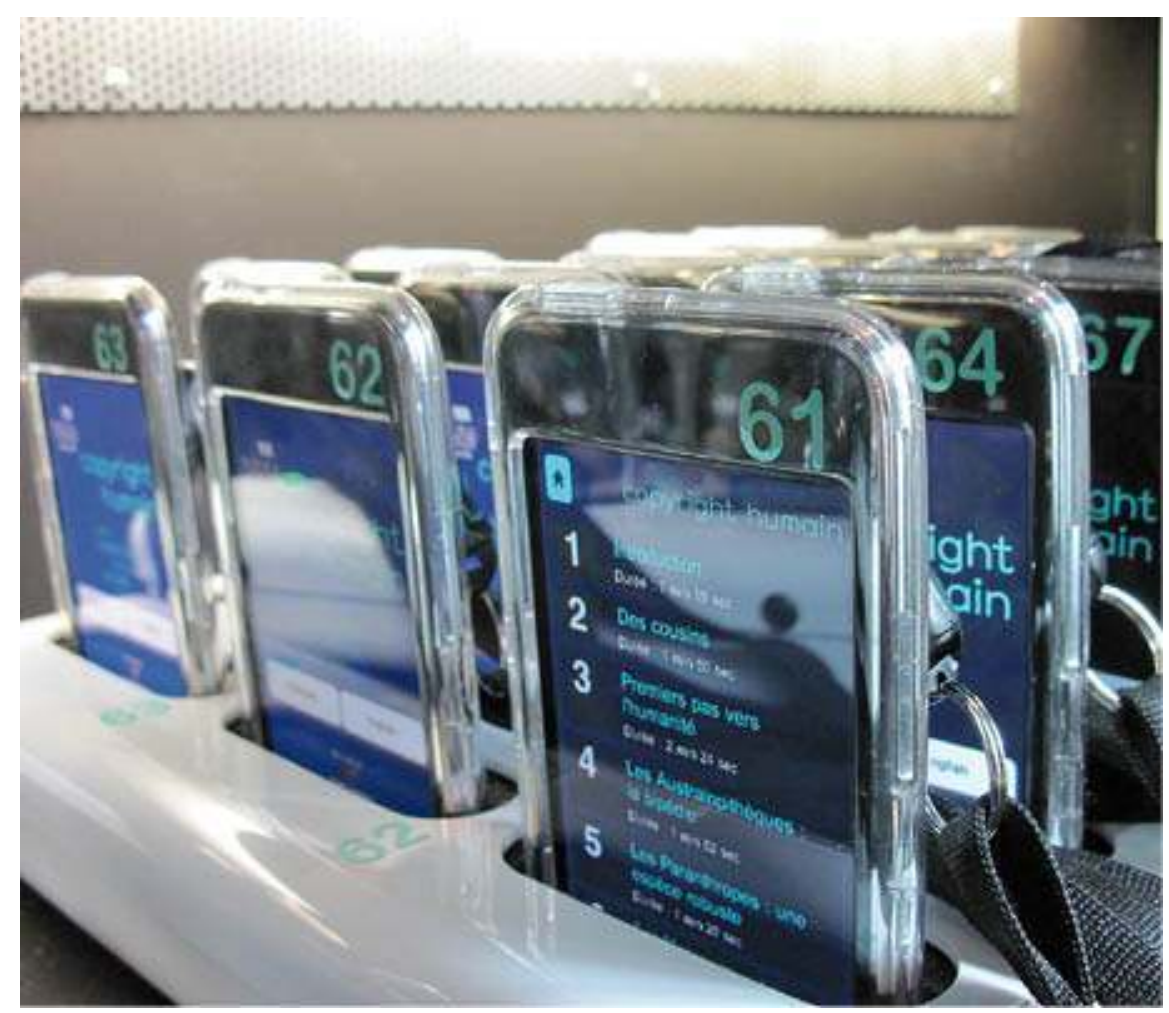

(c) Lucie Daignault 


\section{L'audioguide : un objet et des usages}

6 Les parcours audioguidés ne recouvrent pas tous les mêmes enjeux : un même support (un dispositif audio mobile) peut en réalité servir deux fonctions bien différentes selon les objectifs de conception qui sous-tendent la mise en place de l'audioguide. Les usages ne sont pas les mêmes selon que l'on a affaire à un audioguide conçu comme un outil d'aide à la visite (un support de médiation qui va venir commenter le parcours muséographique) ou à un dispositif sonore intégré à part entière à la muséographie d'un espace de visite ou d'une exposition.

7 Entre ces deux familles de support, la logique de conception est singulière et les logiques de réception adaptatives seront également différenciées. Le public repère et identifie différemment un audioguide dont l'usage s'impose comme une pièce maîtresse de la mise en scène des contenus (en l'occurrence sonore) d'un audioguide proposé comme outil d'aide à la visite et dont l'usage reste facultatif.

8 C'est sur ce type de parcours audioguidés que nous centrerons notre propos - sur la base de résultats d'études et de recherches menées sur des dispositifs technologiques d'aide à la visite (Deshayes, 2009) - en focalisant l'attention sur ce que représente la notion d'outil embarqué pour les publics, soit la plus-value de la mobilité des contenus de médiation en situation de visite.

\section{Expériences de visite et parcours audioguidés}

9 Le public des musées et des expositions est traditionnellement confronté à deux modes de visite possibles : la visite guidée ou la visite libre. Hors le cas des visites sous contraintes, c'est-à-dire en excluant le public dit captif, sur quelle base argumentaire les utilisateurs d'audioguide fondent-ils leur choix pour ce mode de visite?

Il s'agit parfois d'un choix par défaut: la fréquence et le calendrier hebdomadaire des visites guidées ne coïncident pas toujours avec le moment présent d'une visite au musée décidée sans organisation préalable. La contrainte d'un rendez-vous à heure fixe pour la visite guidée n'est pas toujours compatible avec le temps de visite disponible. C'est souvent aussi la contrainte d'une visite guidée en groupe qui fera préférer le recours à l'audioguide : l'image du troupeau de visiteurs agglutinés rebute ceux qui préférent la souplesse de l'audioguide qui permet d'attendre qu'un espace soit désengorgé pour se positionner face à une œuvre...

11 Plus encore, chez certains visiteurs, la métaphore du troupeau n'est pas anodine: elle redouble ici le caractère passif et docile du groupe suivant aveuglement un guide décidant pour tous des moments d'arrêt mais aussi du rythme de la visite.

Avec le guide on est pris par la main, avec l'audioguide au contraire, le visiteur apprécie d'avoir la main: la liberté d'usage qu'autorise la composition d'un numéro d'appel au clavier de l'audioguide est en cela préférée au système d'audioguidage à déclenchement automatique. Quand le parcours est basé sur la technologie infra-rouge par exemple et que ce sont les déplacements des visiteurs qui déclenchent la diffusion des séquences au passage dans une zone de visite, l'écoute du commentaire s'impose à tous et de la même manière. Sur le mode volontariste au contraire, l'écoute "à la demande" donne le 
sentiment de se construire un parcours de visite "sur-mesure", en jouant autant de l'occasion que de la circonstance.

13 Si certains visiteurs auront tendance à écouter tous les commentaires proposés, beaucoup adoptent une stratégie sélective liée à l'attractivité des œuvres ou objets qui reste première. On écoute les commentaires disponibles une fois marqué un temps d'arrêt : la capacité des œuvres ou espaces muséographiques à capter l'attention l'emporte. Le cas échéant, l'audioguide conquièrt une capacité de rétention.

Mais le sentiment de liberté n'est-il pas contraint en réalité par un effet de téléguidage du parcours quand la numérotation dessine un ordre qu'il faudrait suivre? On constate au contraire que les visiteurs s'affranchissent volontiers de l'ordre des séquences proposées. Ils ne suivent pas la numérotation mais ils s'en servent à l'occasion pour se repérer dans l'espace et contrer le sentiment d'avancer en aveugle dans le parcours de visite parfois difficile à anticiper. Sous réserve de certaines conditions, l'usage de tels dispositifs libère le visiteur plus qu'il ne le contraint. Par conditions, on pense en particulier bien sûr à l'ergonomie de l'appareil et à ce qu'il convient d'appeler son "usagibilité", mais aussi et surtout au soin accordé à la signalétique en salle et à son emplacement qui gagnerait à être systématisé. Si le visiteur doit se perdre en repérage et en identification des codes s'y rapportant, l'usage de l'audioguide devient de fait un obstacle à l'optimisation de la visite. À défaut de conditions optimales, il peut rapidement devenir une nuisance (l'utilisateur parle souvent du parcours du combattant pour repérer les numéros de l'audioguide).

Mais pour la plupart des utilisateurs, tout se passe un peu comme si "faire la visite dans l'ordre" était au contraire un contre sens tant le système en soi semble fait pour favoriser une visite à se construire "sur-mesure". La liberté et l'autonomie que confère l'audioguide sont donc interprétées par les visiteurs comme une modalité d'usage : ils s'en saisissent et s'en servent volontiers en ce sens, en dépit d'une autre option possible qui réduirait la visite audioguidée à un parcours dirigé et à une écoute forcée. 
L'audioguide au musée d'Histoire de Marseille : un outil de médiation sonore

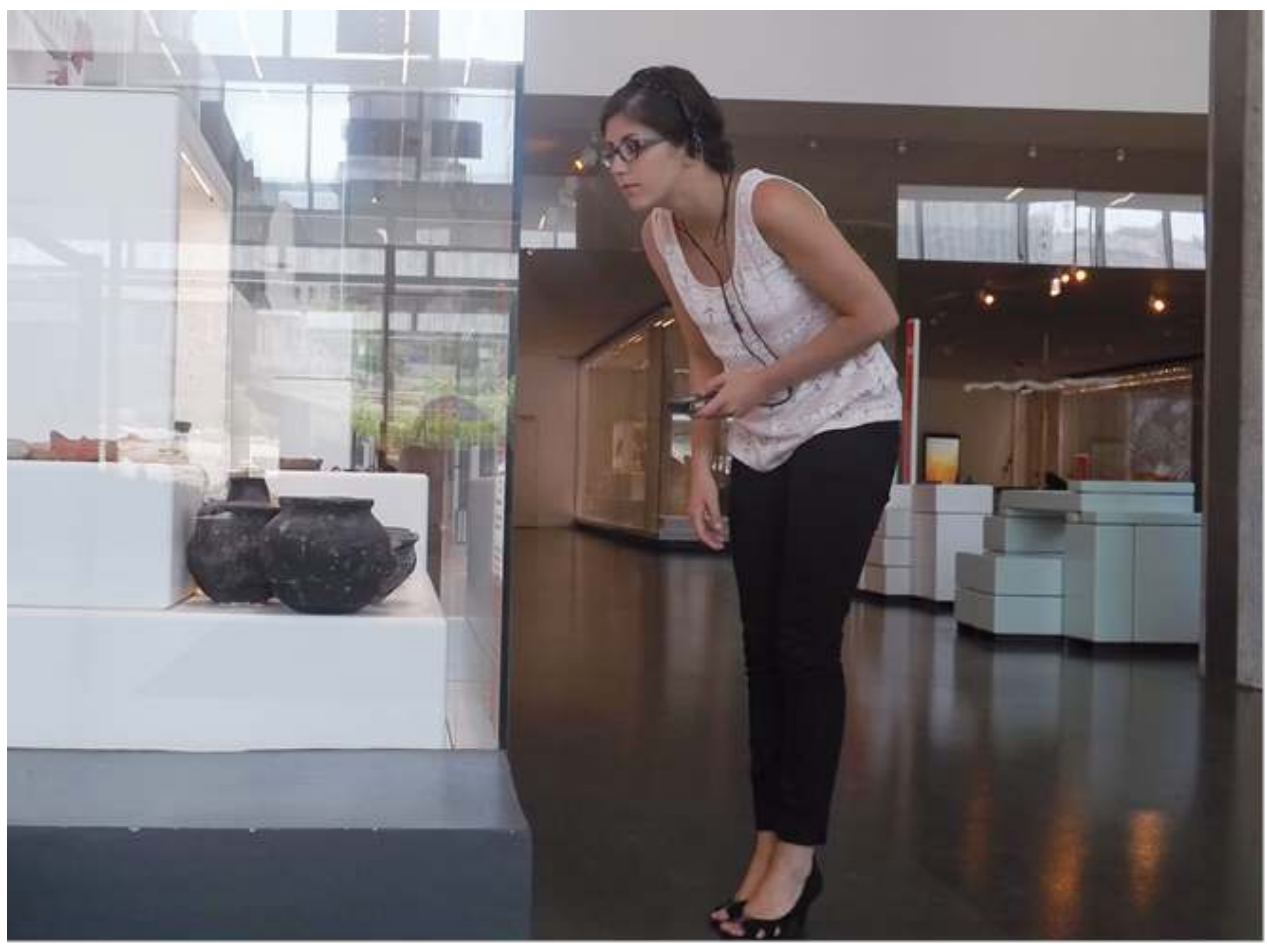

(c) S. Deshayes

\section{Audioguidage, temporalité et sociabilité de la visite}

Les utilisateurs écoutent l'audioguide en utilisant les signaux scriptovisuels que représente la signalétique de l'audioguide, en ragardant les lieux et les objets commentés. À ce titre, l'audioguide, doit plutôt être pensé comme dispositif de communication audiovisuel sous l'angle d'un rapport texte/image (ou si l'on veut écouter/regarder) inscrit non pas dans les propriétés sémiotiques du texte de l'audioguide mais dans son usage. Ce qui fait sens pour les visiteurs utilisateurs, ce ne sont pas seulement les enregistrements audio eux-mêmes, leur inscription sur un support, mais également leurs conditions d'accès en situation de visite.

\section{Écouter et regarder en même temps}

Le support audioguide épouse précisément une dimension propre à la pratique muséale : embarqué, il est aussi mobile que le visiteur qui se déplace dans un musée ou un espace d'exposition.

Par contraste avec la lecture des textes qui diffère (ne serait-ce que de quelques pas) le regard porté aux œuvres ou objets de la collection, ce support de médiation embarqué permet la simultanéité de la contemplation et de l'accès aux clés d'interprétation.

19 Ainsi, l'un des avantages fréquemment avancé de l'usage de l'audioguide est, de fait, la possibilité d'écouter le commentaire tout en regardant l'objet dont il est question. L'audioguide en main et à l'oreille, les visiteurs se déplacent, tournent autour, se baissent à l'occasion, pour affiner le regard tel que le commentaire les y invite. 
nfort d'observation est spontanément comparé à l'alternative statique de lecture des panneaux de textes. Avec l'audioguide, la simultanéité contribue grandement, du point de vue des visiteurs, à l'efficacité du processus : c'est ainsi que certains ont le sentiment de "gagner du temps" dans une logique d'économie du temps de la visite. En réalité, on constate que l'usage de l'audioguide favorise des visites plus longues mais ce vécu d'un gain de temps est ici significatif de l'intensité de l'expérience de visite, d'une dynamique qui s'établit de manière positive et durable.

21

À la différence des pratiques de lecture des textes écrits affichés en salle, la médiation sonore est une proposition temporelle bornée. Les visiteurs doivent généralement renoncer à tout lire, faute de temps et en raison de l'inconfort d'une posture fatigante dans la durée. Pratiquée à l'excès, le lecture devient antinomique de ce pour quoi on est venu : regarder des œuvres, des objets, des mises en scène et non pas lire comme on le ferait tranquillement chez soi, en consultant des ouvrages où les images sont des illustrations des textes. Dans la visite, c'est l'inverse qui opère : les images sont premières, objet de la visite. Les œuvres, collections et espaces scénographiés sont des images réfléchies dans l'œil du visiteur, cherchant dans les textes de médiation à proximité des éléments susceptibles d'éclairer sa perception visuelle.

On constate donc que les visiteurs écoutent et regardent en même temps, non seulement les objets présentés et commentés mais également les cartels associés et les textes de médiation qui se trouvent en salle et dont ils peuvent tenter d'évaluer, de visu, le rapport de contenu avec l'audioguide. Qu'entend-on ici qui n'est pas écrit là ? Convient-il de considérer le statut de ce support comme complémentaire ou en concurrence?

Au musée d'Histoire de Marseille, l'audioguide est aussi utilisé comme complément des panneaux de présentation.

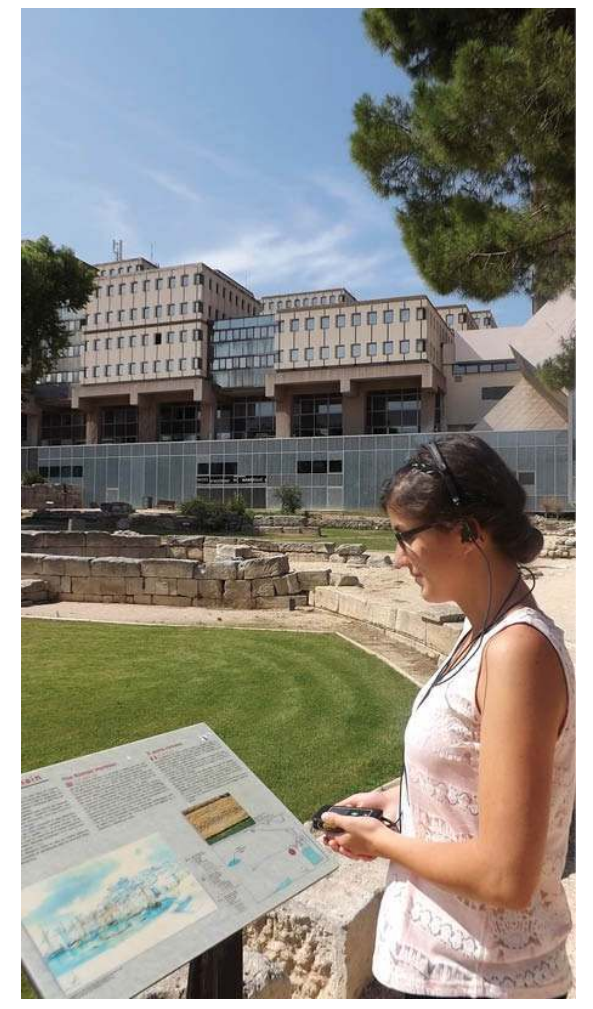

(C) S. Deshayes 
23 À cet égard, la reprise des textes affichés en salle positionne l'audioguide dans un rapport de mise en concurrence, laissée au choix des visiteurs : le contrat de communication est interprété ici comme commodité de confort, une opportunité qui demande un moindre effort : "c'est moins fatiguant d'écouter que de lire".

Mais dans bien des cas, l'audioguide ne reprend que partiellement le contenu des textes affichés, ajoute ou développe certains aspects : à l'usage, les utilisateurs chercheront à déchiffrer un mode d'emploi implicite pour évaluer au mieux les moments où cela vaut le coup de lire et/ou d'écouter... Rapidement comparée aux ressources textuelles, l'écoute des premières séquences est stratégique : elle indique au visiteur le mode d'usage qu'il peut attendre pour en tirer le meilleur bénéfice.

Il serait donc erroné de conclure hâtivement que la médiation audio détourne entièrement les visiteurs de la lecture des textes. L'écoute et la lecture simultanée des titres et en-têtes jouent ainsi souvent fonction de réassurance: si la reprise texto de l'écrit à l'oral est jugée redondante, la reformulation des titres et chapeaux matérialise les sujets abordés, souligne les séquences thématiques associées aux espaces traversés. C'est donc sur un jeu de complémentarité que des usages croisés se mettent en place habitudes de visite, intérêt même de la visite dans le croisement des modes d'approches usages que les visiteurs estiment pertinents et efficaces pour leur propre compte.

À noter que la perception du positionnement éminemment pédagogique de l'audioguide le destine a priori à une plus-value d'accessibilité. Les utilisateurs s'attendent généralement à ce que soit ici explicité le vocabulaire savant et spécialisé souvent méconnu du public non familier des collections ou du monde des musées, celui de la conservation et de la recherche scientifique. Dans le jeu de la complémentarité des supports, les utilisateurs peuvent s'attendre à ce que soit ici vulgarisées des notions inscrites à l'écrit, sur les cartels notamment.

Pour certains professionnels, l'écueil résiderait dans une trop grande attention requise à l'écoute de l'audioguide, la captation de l'attention auditive contrevenant à la disponibilité du corps et de l'esprit. On constate à l'inverse que les visiteurs-utilisateurs ne se sentent jamais prisonniers d'un système qu'ils exploitent à l'occasion fort intensément ou plus distraitement selon les moments de la visite : généralement, c'est à l'occasion de l'écoute de commentaires plus généraux (de type introductif par exemple) que les visiteurs écoutent et regardent en même temps autour d'eux, adoptent une vue d'ensemble, repèrent, observent les autres visiteurs... Le ressort de l'usage s'apparente à la posture d'écoute flottante exploitée en psychanalyse: technique d'appropriation sélective, elle correspond à une posture, intellectuelle et physique, qui rend bien compte de cet état de grande disponibilité où une possible attention diffuse s'avère prompte à capter des messages essentiels.

Du point de vue des visiteurs-utilisateurs, l'écoute flottante de l'audioguide, si elle n'égale pas tout à fait la lecture en diagonale, s'apparente à une stratégie de décryptage efficace. L'usage d'un audioguide autorise une libre déambulation, des moments où l'on écoute et des moments où l'on peut facilement décrocher pour s'installer dans une posture plus contemplative, la souplesse de l'usage des versions numériques étant encore une fois ici attestée. Relevons que rares sont les utilisateurs qui utilisent les boutons de l'appareil prévus pour arrêter le défilement d'un commentaire une fois lancé (touche pause ou stop): si un commentaire intéresse moins, il est plus commode de laisser filer distraitement tout en avançant. 


\section{Parcours de visite audioguidée et sociabilité} autant, les études ont montré qu'il se prête à des usages collectifs dans le cadre des visites où la sociabilité est importante : le plus souvent, on vient au musée en couple, entre amis, en famille. En détournant l'usage strictement individuel de l'appareil, les visiteurs ont tendance à maintenir, voire à alimenter leurs échanges par des conseils d'écoute, l'instauration fréquente du commentaire des commentaires...

Pour cela, l'ergonomie de l'appareil est décisive : les visiteurs préfèrent généralement les audioguides avec écouteurs intégrés préférés aux systèmes avec casques qui enferment davantage dans une bulle sonore qu'il est plus difficile de quitter ou de partager. Les appareils pris en main et simplement posés à l'oreille le temps de l'écoute sont perçus comme une aubaine : il est d'ailleurs fréquent d'observer deux visiteurs intimes écoutant ensemble sur le même appareil.

L'audioguide, outil de sociabilité.

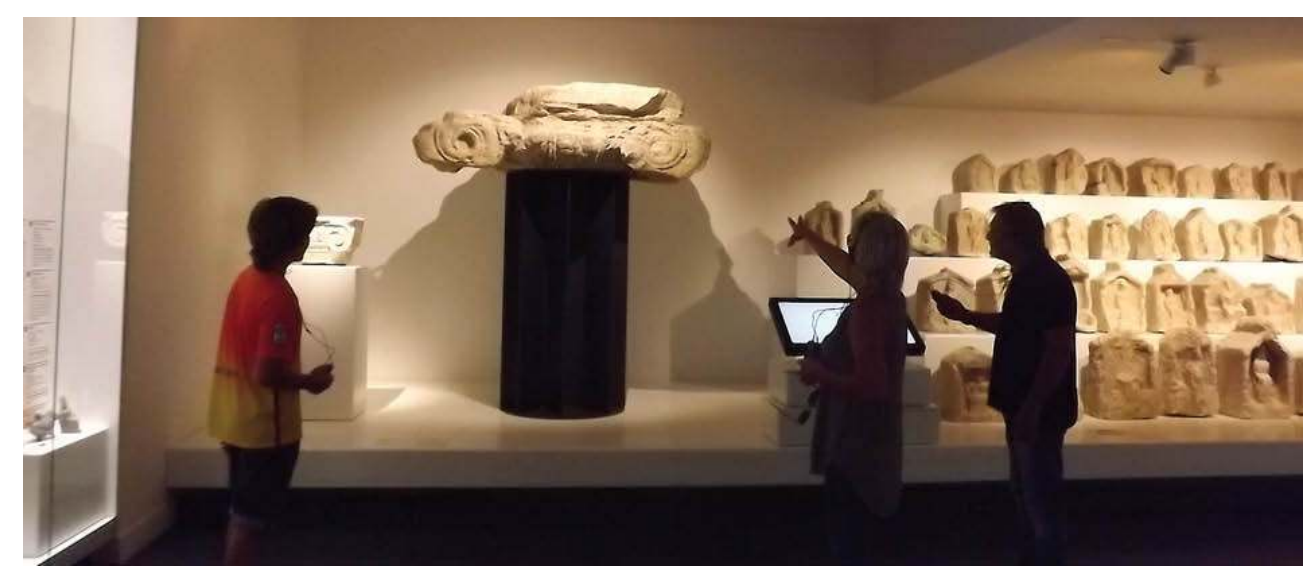

(c) S. Deshayes

À l'inverse, selon des codes de sociabilité préexistant entre visiteurs, l'isolement momentané ou systématique peut au contraire être investi en tant que tel : pour certains adolescents observés visitant en famille, l'usage de l'audioguide apparaît là aussi comme une aubaine. Il permet de s'émanciper du clan, en adoptant un rythme de consultation différent des autres. L'adolescent marque clairement son individualité tout en bénéficiant de la légitimité des bonnes pratiques (écouter l'audioguide est un signe d'attention encouragé plutôt que réprimé). Moins radicale, la logique de l'isolement momentané, le temps d'une écoute librement choisie, permet de visiter à plusieurs sans être continuellement soudés. Dans une salle par exemple, chacun butine à sa guise, avec des moments où l'on se rejoint pour échanger et/ou poursuivre ensemble le parcours. On s'attend généralement avant de changer d'espace de visite, perçu comme une étape, à franchir ensemble pour ne pas risquer de se perdre de vue, gérant ainsi distance et proximité, synchronisation temporelle et continuité de la visite. 


\section{Quand le besoin de parole se fait sentir}

l'autonomie aussi ses limites surtout lorsque l'on n'est pas sufisamment connaisseur pour repérer ce qu'il y a de remarquable. Les publics individuels sont à la recherche de repères qui permettent d'orienter leurs choix : ne pas passer à côté d'un chef d'œuvre sans le voir est ainsi une préoccupation majeure pour les publics non avertis ou peu familiers du musée. Il y a derrière l'idée d'inévitables ratés, "quand on n'y connaît rien". La disponibilité des contenus mobiles est alors un signal tangible exploité en tant que tel. Le support mobile rendant visible - et donc lisible - la présence d'œuvres ou d'objets dignes d'être commentés, son usage constitue un premier critère d'aide aux choix qui assure un minimum de pertinence à la visite. Il s'agit alors de voir (savoir) ce qu'il y a à voir (d'incontournable). L'usage de l'audioguide peut également être anticipé pour décrypter l'implicite d'une mise en exposition. La présence d'un commentaire audioguidé signale au visiteur une hiérarchisation savante, sur la base de critères de discrimination partagés par des spécialistes, des amateurs éclairés, que le visiteur cherche à comprendre.

On constate que de fortes attentes se focalisent ainsi sur la parole, celle de l'institution qui est en jeu : ce qu'elle dit, à commencer par ce qu'elle dit d'elle-même. Les visiteurs savent que les choses ne sont pas là par hasard, qu'il y a un sens, à tout le moins des intentions qui structurent le musée. Ils savent que son organisation par exemple a été pensée, qu'elle reflète une certaine logique - propre à l'institution, à son histoire, à la nature des collections - mais aussi des partis pris. L'usage de l'audioguide est anticipé comme une manière d'entendre le discours de l'institution et à cet égard, accéder à la parole d'un guide incarne l'idée de rencontre, le besoin d'être introduit au musée au sens propre et figuré du terme, ce qu'une approche ouverte de la notion d'accueil devrait prendre en charge. Le même type d'attente introductive se retrouve d'ailleurs à l'entrée d'une exposition, ou d'une salle. Dès que le visiteur pénètre dans une nouvelle zone de visite à part entière, il a besoin d'une mise en condition (de quoi ça parle ici, à quoi ai-je à faire?).

En situation face aux œuvres, certains types de commentaires sont privilégiés, ceux qui éclairent le contexte général qui sous-tend la présentation d'un objet: ce dont il témoigne, l'arrière plan historique ou le contexte d'usage à une époque donnée, un savoir de type ethnologique dès que cela est possible, "la signification, ce que cela pouvait représenter à l'époque" ou encore des éléments de l'histoire de l'art qui permettent de situer l'œuvre dans son contexte. D'autres commentaires sont également appréciés, ceux qui donnent le sentiment d'apprendre à voir un détail, un aspect que l'on n'aurait pas remarqué sans cela.

Quand les visiteurs disent que l'audioguide leur permet d'apprendre des choses au musée, et en particulier d'apprendre à voir, ce n'est pas seulement la vision de l'œuvre qui s'enrichit. Dans la parole, la voix, savoir et savoir-faire sont extrêmement mêlés et certains commentaires provoquent ainsi une double révélation: les visiteurs voient des choses qu'ils n'auraient pas forcément remarqués par eux-mêmes mais ils voient aussi la manière dont des spécialistes s'y prennent pour regarder, les façons de faire qui permettent aux connaisseurs de discriminer, d'observer en portant un regard avisé. L'usage de l'audioguide donne ainsi le sentiment d'acquérir une compétence, celle de l'attention'. 
Des pictogrammes au sol dirigent les utilisateurs de l'audioguide au musée des Instruments de Musique de Bruxelles.

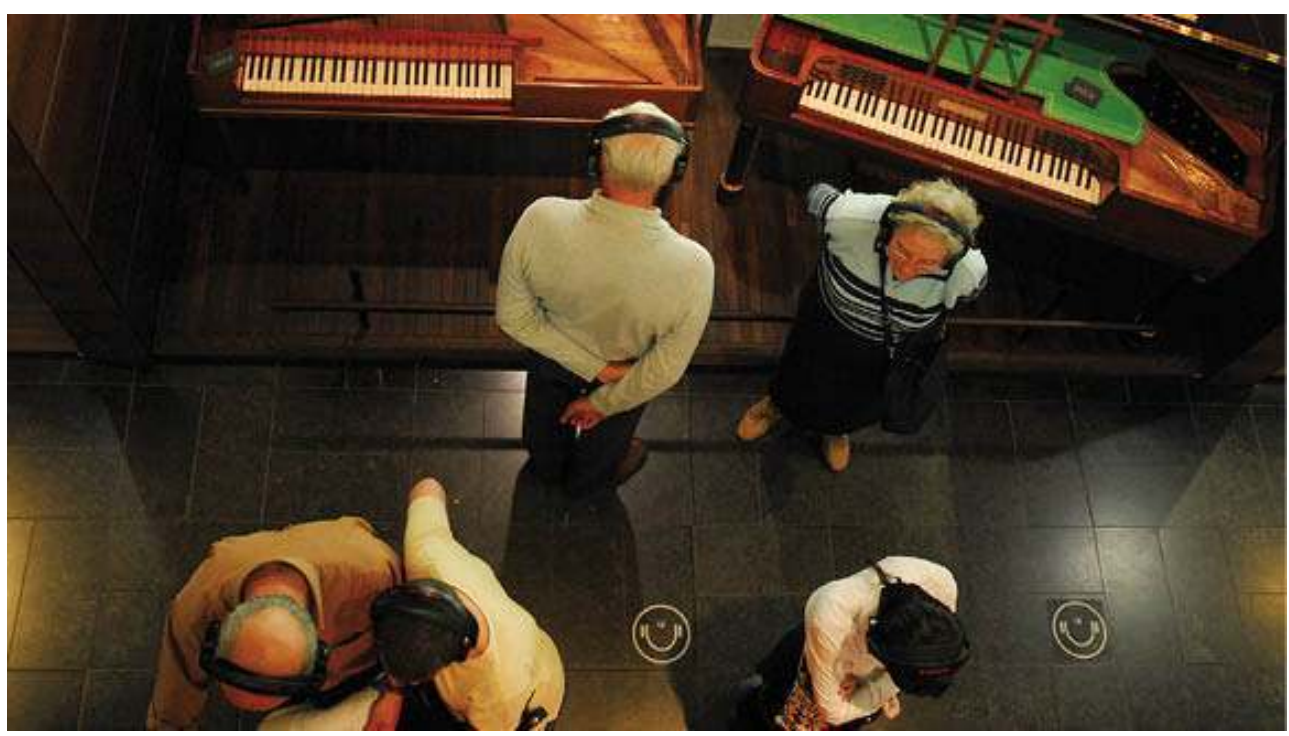

(c) MIM

\section{L'importance de la qualité de l'énonciateur}

Établies du point de vue des publics, nos analyses ont mis à jour un horizon d'attente resté longtemps sous-exploité dans la conception des parcours audioguidés, celui de l'anticipation d'un mode de relation au savoir par l'écoute de paroles incarnées.

Par l'entremise de la voix qui parle, c'est la parole d'un guide qui est attendue, sur le mode de quelqu'un qui raconte, souligne, pointe, suggère, sous-entend... L'intérêt de l'écoute réside autant dans les connaissances transmises par quelqu'un qui sait que dans la manière de le dire. C'est la voix de quelqu'un qui est en train d'utiliser son savoir en même temps qu'il le transmet. L'intérêt de l'écoute, c'est la parole de quelqu'un qui sait, la voix d'un spécialiste (dont les savoirs sont construits), celle d'un amateur éclairé, d'un médiateur averti ou la figure d'un grand témoin connaisseur.

En réponse à cet horizon d'attente de paroles savantes vives et incarnées, dès 2003, nous avons préconisé de concevoir la médiation audio sur la base de modèles plus radiophoniques que littéraires: la communication des savoirs gagne à s'affranchir de textes écrits pour être lus (fussent-ils correctement "oralisés"). L'audioguide s'éloigne désormais des enregistrements d'une voix off anonyme, et en cela correspond bien davantage aux attentes des publics. En 2007, lorsque le premier audioguide multimédia du musée du Louvre renouvelle entièrement la conception des programmes diffusés, cette approche encore inédite pour les utilisateurs familiers des contenus standards, est tout particulièrement soulignée et plébiscitée par les publics usagers ${ }^{2}$. Basé sur l'enregistrement de conservateurs et de spécialistes, propos captés à travers des situations face aux œuvres, l'intérêt des publics pour ces contenus perdure ${ }^{3}$.

Lorsqu'elles accompagnent, en le favorisant et en l'intensifiant, le goût des savoirs, les technologies deviennent de puissants leviers d'acculturation, des outils culturels éducatifs au sens plein du terme. Les difficultés actuelles résident dans la capacité à 
résister aux seules tentations offertes par les constructeurs promoteurs de ces nouvelles technologies, aux stratégies marketing qui souvent imposent des fonctionnalités nouvelles, en dépit de l'appauvrissement de la qualité de la médiation espérée. Ainsi, il est symptomatique que les scénarios contributifs - voir par exemple, la présentation de résultats d'enquêtes menées par le musée du Louvre (Krebs, 2013) - soient si peu investis par les publics en situation de visite - résultat récurrent des études de réception manifestement peu connu des promoteurs de l'avènement des cultural games qui ferait vendre : l'actuel audioguide multimédia Nintendo au Louvre en est un bon exemple.

Si, à l'inverse, les technologies réactualisent le désir d'être en contact avec des gens du musée, des spécialistes ou des guides conférenciers passionnés ${ }^{4}$, quelque chose de l'ordre de la transmission passe et se passe au musée. Ainsi, le succès d'estime dont bénéficie le nouveau parcours permanent des collections du musée d'Histoire de Marseille doit beaucoup à la place réservée aux paroles de spécialistes grâce aux écrans multimédia - à leur statut de grands témoins - qui rythment le parcours, accompagnent la déambulation au musée, enrichissent l'attention que l'on porte aux objets des collections.

\section{BIBLIOGRAPHIE}

Chaguiboff, J. et Matharan, J. Les premiers pas du guide multi-média. Rapport d'évaluation, Cabinet Plein Sens, musée du Louvre, janvier 2008.

Daignault, L. et Cousson, C. Quand la technologie s'invite au musée, La Lettre de l'OCIM, $\mathrm{n}^{\circ} 137$, septembre-octobre 2011, pp. 5-11.

Deshayes, S. Audioguides et musées, La Lettre de l'OCIM, nº 79, janvier-février 2002, pp. 24-31.

Deshayes, S. L'usage des supports mobiles au musée : des audioguides classiques au multimédia nomade, in Actes du colloque ICHIM Berlin, août-septembre 2004, CDROM édité par Xavier Perrot, université Paris 1 Panthéon-Sorbonne.

Deshayes, S. Nouvelle génération d'audioguide : démarche de conception et choix du multimédia mobile au museon Arlaten, La Lettre de l'OCIM, n 92, mars-avril 2004, pp. 17-23.

Deshayes, S. Amour de l'art ou amour des savoirs ? Exemple d'une recherche-développement pour la conception d'un dispositif de visite urbaine sur téléphone mobile, in Actes du XIXe Congrès de l'Association Internationale d'Esthétique Empirique, université d'Avignon, 29 août-1er septembre 2006

Deshayes, S. Les sciences humaines et sociales s'exposent : expérience de visite et actualisation des savoirs à l'Historial de Péronne, Culture \& Musées, n 10, Actes Sud, 2006.

Deshayes, S. Savoirs et paroles médiatisées au musée. Analyse communicationnelle de l'audioguidage. Thèse de doctorat, École Normale Supérieure Lettres et Sciences humaines, laboratoire Communication Culture et Société, Lyon. École doctorale Éducation - Psychologie Information et Communication, 2009.

Dor, A. Les audioguides... ont de l'oreille ! La Lettre de l'OCIM, $\mathrm{n}^{\circ} 120$, novembre-décembre 2008, pp. 11-19. 
Krebs, A. Numérique et institutions culturelles : quelle création de valeur ? Conférence plénière, Rencontre Médiation \& numérique dans les équipements culturels, BNF, mardi 22 octobre 2013, www.rencontresnumeriques.org/2013/mediation/ ?action =restitution.

Mœglin, P. Outils et médias éducatifs. Une approche communicationnelle. Presses Universitaires de Grenoble, 2005.

Perriault, J. La logique de l'usage : essai sur les machines à communiquer. Paris : Flammarion, 1989.

\section{NOTES}

1. Certaines conditions énonciatives doivent pour cela être respectées. Certains publics s'avouent ainsi quasiment "allergiques" aux audioguides car trop injonctifs Voir nos premières contributions sur le sujet La Lettre de l'OCIM, n79, 2002 et n92, 2004.

2. Chaguiboff, J. et Matharan, J. (2008). Données d'évaluation qui viennent corroborer les résultats préexistants sur cet aspect essentiel de la communication expérimenté dans le cadre du projet EECCOOT, programme de recherche-développement qui, de 2003 à 2006, a associé une société privée (Tout O Phone), des équipes de recherches en sciences sociales (C2SO, ENS-LSH), en informatique (CITI, INSA) et en économie (LET, ISH), des partenaires culturels (musée Gadagne à Lyon et Office de tourisme intercommunal Nord de la Réunion), pour créer un dispositif de visite culturelle par téléphone portable (Deshayes, 2006).

3. À l'inverse, en dépit des pronostics marketing, une plus grande appétence pour l'audioguide Da Vinci Code n'a pas fait ses preuves.

4. L'écoute d'extraits d'interviews ne convient pas cependant à toutes les générations de publics : les résultats d'une étude formative que nous avons conduite au musée d'Histoire de Marseille pour la conception du programme audioguidé destiné aux adolescents a démontré leur préférence pour une scénarisation sous une forme dialoguée éloignée de la figure professorale. Cette étude, qui n'a pas encore été publiée, a été conduite en collaboration avec la société Audiovisit en 2013.

\section{RÉSUMÉS}

L'analyse des pratiques des publics lors de visites audioguidées permet de constater qu'en plus de la liberté et de l'autonomie que confère l'audioguide, le visiteur attend de l'usage de cet instrument de médiation un nouveau mode de relation au savoir dispensé par l'institution muséale.

INDEX

Mots-clés : audioguide, médiation sonore 


\section{AUTEUR}

\section{SOPHIE DESHAYES}

docteur en Sciences de l'Information et de la Communication et responsable de la programmation culturelle au musée d'Histoire de la ville de Marseille

sophie.deshayes@chadocs.com 